\title{
What Priming Techniques Can Tell Us about Associative Representations Acquired During Human Contingency Learning
}

\author{
Joaquín Morís*, Pedro L. Cobos and David Luque \\ Departamento Psicología Básica (Facultad de Psicología), Universidad de Málaga, Campus de Teatinos, Málaga \\ 29071, Spain
}

\begin{abstract}
Associative theories of learning have been used to explain human contingency learning since the 1980's. Recent findings have led several authors to claim that there is no evidence clearly showing the engagement of associative processes of acquisition or representation in human contingency learning, and to propose non-associative accounts. Priming techniques can detect associative representations when the right parameters are employed. The present paper reviews evidence available of associative representations created after human contingency learning obtained using priming techniques. The evidence reviewed supports associative theories of learning and the assumption of spreading activation and associations between representations.
\end{abstract}

Keywords: Human contingency learning, blocking, priming, memory, learning.

\section{OVERVIEW}

Human contingency learning (HCL) theories try to explain how humans learn the relations between the presence and absence of some cues and the presence or absence of relevant outcomes, and how this learning guides their later behaviour. There are a large number of models and theories that try to account for HCL and propose very different learning mechanisms and representations of the knowledge acquired. Until recently, the most accepted point of view was that both associative and higher level processes, like reasoning, could be involved in HCL. But this has now been questioned by several authors (for reviews see [1-3]). Most of the discussion has focused on the nature of the processes engaged in acquisition during HCL. The purpose of the present work is to review the evidence available of the existence of associative representations in HCL, and its theoretical relevance.

\section{ASSOCIATIVE THEORIES AND THEIR DOMINANT POSITION IN HCL}

In the 1980's several works led to a great growth of the study of HCL, as they related human learning to animal associative learning and the group of theories and models used to explain it (for a review see $[4,5]$ ). For example Dickinson, Shanks and Evenden [6] found evidence of blocking, a phenomenon described earlier by Kamin in rats that later proved to be critical for theories of animal learning. The blocking phenomenon, which is a kind of cue competition effect, is observed when participants are exposed to Cues AB-Outcome 1 pairings after having been exposed to

*Address correspondence to this author at the Departamento Psicología Básica (Facultad de Psicología), Universidad de Málaga, Campus de Teatinos, Málaga 29071, Spain; Tel: +34 952 132630;

Fax: +34 952 132631; E-mail: jmoris@uma.es
A-1 pairings. In such circumstances, participants' judgments about the B-1 relationship are lower than if they had not been exposed to A-1 pairings. Shanks [7] showed that humans' verbal judgements were sensitive to contingency manipulations in a similar way as responses of non human animals (e.g. $[8,9])$. These findings strengthened the associative account of HCL.

\section{MAIN ASSUMPTIONS OF ASSOCIATIVE THEORIES}

Associative theories share a common set of assumptions that were unchallenged for a very long time. We will use the allergies task to exemplify them. The allergies task, or versions of it, has been widely employed in HCL studies and has become paradigmatic (e.g. [10-13]). In its most common version, participants are told that they will play the role of a doctor. They see what types of food a patient has ingested and they have to predict if he is going to suffer an allergy or not. As trials pass, participants learn the different relations between the cues used and the presence or absence of allergy in the patients, and later, in a test phase, are asked to what degree they think that a given cue, or food, is responsible for the allergy. In a broad sense, according to associative theories the presentation of cues and outcomes are processed by an associative mechanism that updates the strength of the associations between their representations. This mechanism is thought to be automatic, non strategic, and capable of working relatively unaffected by cognitive load and topdown processes of higher order (e.g. [10]). Many different models have been proposed at a computational and algorithmic level for both the mechanisms and representations involved (e.g. $[8,14,15])$. The presentation of a cue would activate its representation, and through its associations, that activation would spread to the representations of the outcomes. In the case of verbal judgments, it was supposed that the judgement given would reflect the strength of the excita- 
tory or inhibitory association between the cue and the outcome (e.g. [7]).

\section{PROBLEMS OF THESE ASSUMPTIONS-EVIDENCE FOR THE ROLE OF OTHER PROCESSES}

Associative theories have not been the only ones developed to explain HCL. Inferential theories have been some of the most successful (see [4] for a review). Among the common features that they share it is especially relevant for this review that all of them have a non-associative view of the representation of knowledge. For example, some of them state that raw frequency of events will be stored and later used to compute conditional probabilities (e.g. [16]), others propose that humans create propositions that can later be evaluated given the information available on test trials (e.g. [1]).

Recently, further research inspired by non associative theories of HCL has shown that associative processes might not be the only ones involved in HCL, and have even questioned the existence of associative mechanisms or representations (for reviews, see $[1,2,17])$. A great deal of the experimental evidence comes from blocking experiments as blocking, and cue competition in general, has served as the main arena in which associative and non-associative theories have disputed their prevalence in explaining HCL. Among the most recent blocking phenomena that have challenged the associative framework, we will focus on the additivity and type of question effects, the dependence of blocking on the flexible use of information, and the influence of overloading secondary tasks on blocking.

\section{Additivity and Maximality Training}

In a blocking design, there are two training phases: a first one consisting of $\mathrm{A}+$ trials (i.e., trials in which Cue $\mathrm{A}$ is followed by the outcome), and a second one consisting of $\mathrm{AB}+$ trials. Adding the first phase causes a decrease in the conditioning of Cue B [18] or in participants' judgments on the relationship between Cue B and the outcome. According to associative models based on error correction algorithms, like Rescorla and Wagner's [8], the presentation of the outcome is fully predicted by cue A, therefore nothing will be learned about cue B. Lovibond, Been, Mitchell, Bouton and Frohardt [19] proposed a different approach to the same phenomenon. They hypothesized that participants might be using reasoning processes in HCL tasks. Therefore, participants might reason that given that the presence or absence of $\mathrm{B}$ does not alter the consequences that follow the presentation of cue A, $B$ is not an underlying cause of the appearance of the outcome. According to this, if B were also a cause of the outcome, the magnitude or probability of the outcome should be higher. According to causal reasoning theories, if the outcome is binary (it can only be present or absent), and its probability of occurrence given A is 1 , the reasoning processes could be hindered, reducing the size of the blocking effect. This is because, even if B is causally related to the outcome, it will not raise the probability or the magnitude of the outcome, as it already occurs at its maximum magnitude or probability given the presence of A. To test this, Lovibond et al. [19] used a design with two groups that received a different pretraining. For the first one, or nonadditive group, the outcome was binary, and participants were presented with
$\mathrm{J}+, \mathrm{K}+$ and $\mathrm{JK}+$ trials. For the second, or additive, group, the outcome could have two different values. Specifically, on J+ and $\mathrm{K}+$ trials, the outcome had the lowest value, whereas, on $\mathrm{JK}++$ trials, the outcome had the highest value. Later on, both groups went through the same $\mathrm{A}+\mathrm{AB}+$ training phases of a typical blocking design. As a result, the blocking effect was higher in the additive group than in the nonadditive group, which the authors interpreted as evidence in favour of an inferential account of blocking. This effect and related ones have been replicated with several procedures and also in non human animals [20-24]. Beckers, De Houwer, Pineño and Miller's [20] Experiment 4 is specially compelling, as the design is equivalent to the one discussed before except for the fact that the pretraining phase took place between the blocking trials and the test. The results were nonetheless the same, i.e., an increased blocking effect in the additive group. Although some associative accounts of this effect have been proposed $[12,25]$, they currently seem to lack the power to explain all of the evidence available.

\section{Secondary Task}

Some non-associative theories of HCL conceptualize learning as a controlled, conscious, slow and effortful process (e.g. [17]). This contrasts with the view of associative learning processes as automatic, rapid, and effortless. De Houwer and Beckers [26] introduced a secondary task in a blocking experiment. According to their hypothesis, the secondary task should produce a high cognitive load which, in turn, would prevent the reasoning processes to fully operate, reducing, thus, the blocking effect. On the other hand, if associative processes were responsible for blocking, cognitive load should not produce any change in the magnitude of blocking. Consistently with their hypothesis, in their Experiment 2 , they found a smaller blocking effect in a group that performed a difficult secondary task during the learning and the test phases than in another group that performed an easier secondary task. Interestingly, in Experiment 1, in which the secondary task was performed only during the learning phase, no differences in blocking were found between the groups. The authors claimed that those reasoning processes responsible for, at least, part of the blocking effect could have been working during learning. But those participants who were prevented from engaging in reasoning processes during that time could have been doing it later during the test phase. The effect of a secondary task on blocking was later replicated by Vandorpe, De Houwer and Beckers [27].

\section{Type of Question and Flexible Use of Information}

As stated above, from an associative point of view, the verbal judgement used as the dependent measure is proportional to the strength of the association between the cue and the outcome. However, several studies have shown that this assumption might not be completely correct. For example, Price and Yates [28] used different test questions in a cue interaction design that could be considered, in essence, a blocking design. They observed the blocking effect with causal and conditional probability questions, but not when participants judged the frequency of cooccurrence of cues and outcomes. Matute, Arcediano, and Miller [29] found similar results with another cue interaction design. Addition- 
ally, other studies have found robust effects of type of question on participants' judgments by using very different non-cue interaction designs. Specifically, interference and contingency manipulations have been shown to differently influence participants' judgments depending on whether the questions used were predictive, causal or preparatory [30-32].

The activation-spread assumption of associative theories is, clearly, too limited to account for the whole pattern of results concerning the influence of type of question. This consideration has led some researchers to claim that the information is stored in a rather 'raw' manner throughout the training trials, allowing for later flexible use performed by rule-governed or inferential reasoning processes (see [1]). In any case, as Vadillo, Miller and Matute [32] have pointed out, though these results are not completely incompatible with associative representations or acquisition mechanisms, another postacquisition process would be required in order to flexibly use the information available to the subject.

These and other findings gave a strong support to the idea that associative processes are unlikely to be the only ones engaged in HCL. But for some authors this is a mild view of the problem. For example De Houwer, Beckers and Vandorpe [17] state that "To conclude, we believe that there is strong evidence to support the hypothesis that cue competition effects are due either to the fact that participants make an inference about a cue-outcome relation on the basis of available conscious propositional knowledge or to the fact that they do not pay attention to cues that are always presented in compound with other cues that have previously been established as causes or predictors of the outcome" ([17], p. 246). Also, Mitchell, Lovibond and De Houwer [2], given the evidence showing that verbal instructions about the relations between stimuli seem to produce the same behavioural results as repeated experience with those stimuli (e.g. [33]), claim that "This shows that knowledge acquired verbally and knowledge acquired by direct experience is represented in a similar way. Hence, the implication is that the knowledge acquired by experience is propositional in nature" ([2], p. 190).

Thus, both reviews conclude that the best way to conceive learning is by invoking reasoning processes that operate upon propositional representations of knowledge, and that associative processes and representations do not play any role in HCL. Given this state of affairs, we wonder whether this hard claim that associative processes have a null explanatory role in HCL is actually tenable. As a first step to answer this question, we could search for more direct evidence supporting the assumptions from the associative framework. According to them, the experience with cues and outcomes produces associations between the representation of the cues and the representation of the outcomes. At test, the activation spreads from the representation of the presented cue to the representation of its associated outcome. Thus, could we find any evidence of this activation spread? If so, does the evidence of activation spread behave like verbal judgments regarding relevant learning phenomena as blocking?

From an associative point of view, the repeated presentation of a cue followed by an outcome should produce an activation spread from the representation of the cue to the representation of the outcome when the cue is presented at test. Additionally, if a cue undergoes a blocking treatment, the activation of its representation should spread to a lesser extent than the representation of a control cue not subjected to a blocking treatment. Though these are predictions directly derived from core assumptions within the associative framework, they have not been tested yet. Is there any evidence consistent with these predictions?

\section{WHY PRIMING?}

Priming techniques have been widely used in the study of memory and psycholinguistics, and it is well known that using adequate parameters, associations between representations of stimuli can be measured without the engagement of high level or strategic processes [34-37].

Priming can be defined as a variation in the processing of a target stimulus due to the previous presentation of a priming stimulus. It can be facilitatory, when the target is processed faster and with fewer errors, or inhibitory whenever the processing speed is reduced or the number of errors is increased. One classical example of priming is semantic priming, a type of priming that takes place when priming and target stimuli are semantically related. During a lexical decision task, participants have to decide if a given letter sequence is a correct word, like tiger, or a pseudoword, like tegir. Presenting a priming stimulus semantically related to tiger, for example lion, will produce a reduction in the time needed to correctly identify tiger as a word compared to another situation in which it is preceded by another non related stimulus, for example loin. This happens even when prime and target pairs throughout the lexical decision task are arranged so that primes provide no information about what target will appear. For example, given a prime word, a target word will follow in $50 \%$ of the cases. And, in such cases, only a small percentage of pairs will be formed by semantically related words. This same effect has been found using other tasks, like naming or stem completion, and under different situations (e.g. [38, 39]). Another kind of priming, in which we will focus later, is associative repetition priming. When pairs of words are studied together, the presentation of one as a prime will produce a facilitated processing of the other, as it has been found in word stem completion [40], lexical decision (e.g. [41]) and perceptual identification [42]. As in HCL, relationships between stimuli are learned in a first phase, and then they are tested later.

\section{UNDER WHAT CONDITION DOES PRIMING PREVENT STRATEGIC PROCESSES?}

One critical parameter in priming tasks is the Stimulus Onset Asynchrony (SOA), i.e., the time elapsed between the onset of the prime and the onset of the target stimulus. There is ample evidence both from behavioural and neuroimaging studies showing that the use of short SOAs (300ms or less) can reduce the influence of high order and strategic processes to a minimum (e.g., [34, 36, 43]). Thus, priming effects observed at short SOAs are likely to reflect the operation of automatic retrieval processes as those proposed by associative theories. Furthermore, spreading-activation theories (e.g., [44-46]), which are the most widely accepted explanations of priming, propose representations and retrieval processes similar to those proposed by associative theories of 
learning. Although they differ in many aspects, they share common ideas that are central to them [47]: (1) these theories conceptualize representations as nodes that can be linked or not, forming a network, (2) the activation of one of these nodes will produce some spreading of that activation to those nodes linked with it, but not to nodes not connected to it, (3) in order to retrieve an item, a given amount of activation of its internal representation has to be reached, (4) residual or previous activation of the representation will facilitate reaching the threshold level of activation and therefore its retrieval. Also, this would happen in a short time, and in an unintentional and potentially unaware way (e.g. [48]). According to these theories, presenting a prime will activate the representation node for that stimulus, and some of that activation will spread to the representation of the target. Due to this activation prior to the presentation of the target, less time will be required for the corresponding node to reach its threshold once the target is presented, which would explain the priming effect.

\section{RATIONALE}

Therefore, associative repetition priming experiments seem to be the best candidate to solve the question that was posed before, does HCL lead to the creation of associative representations of the relations learned and do these representations conform to what it is expected according to associative theories? We will now review several associative repetition experiments that provide relevant information.

Soon after the discovery of semantic priming [49], McKoon and Ratcliff [41] provided the first demonstration of associative repetition priming as part of a broader research project about semantic and episodic memory. According to some theories (e.g. [50]), episodic and semantic memory are different memory systems. McKoon and Ratcliff wanted to test if episodic experience affects participants' responses in a lexical decision task, which was considered to involve semantic processes. Table 1 shows part of the design used in Experiment 1. First, participants went through a study phase during which they were presented with pairs of words. Each one was presented once for 3 seconds. In the conditions we are interested in, these pairs were formed by words that had no semantic or other preexperimental relation. For example, participants could see the pair of words city and grass together for 3 seconds, then the pair marble and home, then lovely and sour, and so on. They were instructed to learn all the pairs for a later memory test. Subsequently, participants faced a lexical decision task in which they had to indicate whether each of a series of letter strings, or targets, was a word or not. Each target was preceded by a prime consisting of a word. Prime-target pairs were arranged according to different conditions. In the coherent condition, the prime and the target had been previously paired in the study phase (e.g., city-grass), whereas, in the recombined condition, the prime and the target had been presented as constituents of different pairs during the study phase (e.g., marble-sour). McKoon and Ratcliff found faster lexical decision responses in the coherent than in the recombined condition, i.e., they found a facilitatory priming effect when primes and targets had been previously associated (see Table $\mathbf{2}$ for the size of the priming effect of this and the rest of priming experiments discussed). They interpreted this finding in terms of an activation spreading mechanism. But this claim was challenged later by other authors like Carroll and Kisner [51] or Durgunoglu and Neely [52]. They pointed out that, since participants were also required to give a response to the prime in some of McKoon and Ratcliff's [41] experiments, the time between the onset of the prime and the onset of the target (the stimulus onset asynchrony, or SOA) was long enough to allow participants to use expectancy-based strategies. Briefly, when participants were presented with the prime, they could have retrieved the associated target. Thus, the facilitatory priming observed could have been due to the match between the target presented later on and the memory previously recalled, rather than to an automatic activation spreading process. It has to be noted that McKoon and Ratcliff's [41] Experiment 3 , dealt with this problem by using a fixed SOA in a lexical decision task in which no response to the prime was required. Again, a reduced response time for previously associated prime-target pairs compared to the recombined controls was found even when short SOAs (e.g., $300 \mathrm{~ms}$ ) were used, thus preventing the intervention of controlled processes as those involved in expectancy-based strategies (see also [53]). Equivalent results have been found using different procedures and parameters (see [37] for a review).

Table 1. Design of Experiment 3 by McKoon and Ratcliff [41]

\begin{tabular}{|c|c|c|}
\hline \multirow{2}{*}{ Training } & \multicolumn{2}{|c|}{ Test } \\
\hline & Coherent & Recombined \\
\hline \hline $1 \times A 1$ & $\mathrm{~A}-1 ?$ & $\mathrm{~F}-1 ?$ \\
\hline $1 \times \mathrm{x} 2$ & $\mathrm{~B}-2 ?$ & $\mathrm{E}-2 ?$ \\
\hline $1 \times \mathrm{C} 3$ & $\mathrm{C}-3 ?$ & $\mathrm{D}-3 ?$ \\
\hline $1 \times \mathrm{DD} 4$ & $\mathrm{D}-4 ?$ & $\mathrm{C}-4 ?$ \\
\hline $1 \times \mathrm{xE} 5$ & $\mathrm{E}-5 ?$ & $\mathrm{~A}-5 ?$ \\
\hline $1 \times \mathrm{xF} 6$ & $\mathrm{~F}-6 ?$ & $\mathrm{~B}-6 ?$ \\
\hline
\end{tabular}

Note: Letters A-F stand for cues, and numbers 1-6 stand for outcomes.

Table 2. Size of the priming effect in McKoon and Ratcliff [41] and Morís et al. [54]

\begin{tabular}{|c|c|c|}
\hline McKoon and Ratcliff [41] Exp. 1 & & 40ms* \\
\hline \hline $\begin{array}{c}\text { McKoon and Ratcliff } \\
\text { [41] Exp. 3 }\end{array}$ & $70 \mathrm{~ms}^{*}$ \\
\hline $\begin{array}{c}\text { Morís } \text { et al. [54] } \\
\text { Acquisition experiment }\end{array}$ & $50 \mathrm{~ms}$ \\
\hline $\begin{array}{c}\text { Morís } \text { et al. [54] } \\
\text { Blocking experiment }\end{array}$ & $\begin{array}{c}\text { Blocking condition } \\
\text { Control condition }\end{array}$ & $\begin{array}{c}6 \mathrm{~ms} \\
81 \mathrm{~ms}\end{array}$ \\
\hline
\end{tabular}

Note: The size of the priming effect is the mean reduction of the RT to the target when it was primed by a stimuli with which it was paired during training compared to its proper control. An asterisk indicates that the effect was significant.

Therefore, these experiments have shown that associative repetition priming can be used to detect the associations formed between stimulus representations. However, it is one 
thing to provide evidence of associative memories after experience with events, and another thing altogether to show that such associative memories have something to do with HCL. On the one hand, the evidence coming from HCL, as well as from animal conditioning, tell us that the learning of relationships between events does not merely consist in recording memories of experienced cue-outcome occurrences. Results from blocking and, more generally, cue competition experiments support the view of learning as a process designed to infer predictiveness relationships on the basis of the amount of information about the occurrence of outcomes conveyed by cues. Thus, it is crucial to use blocking (or cue competition) designs in associative repetition priming experiments to find more compelling evidence for the relationship between the facilitatory priming effects in associative repetition priming and HCL. It is also important to remember that, as we said above, the blocking effect is an important focus of interest in the debate between associative and nonassociative theorists of HCL. On the other hand, there are some procedural differences between HCL and associative repetition priming experiments that could be relevant for the priming effects found. For example, the frequency of each stimulus pair in the study phase of associative repetition priming experiments is usually lower than the frequency of each cue-outcome pair in HCL experiments. Also, in associative repetition priming experiments, participants are usually instructed to learn lists of words, whereas, in HCL experiments, participants usually have to predict what outcome will appear following the cue or cues present on a given trial.

To fill this gap between HCL and associative repetition priming, Morís, Luque, Cobos and López [54] conducted, among others, two experiments, one using a blocking design that included a test phase based on priming instead of verbal judgments. The first one was designed to validate the task, with the aim to replicate previous experimental results with the task that will be described. As expected, a facilitatory priming effect was found (see Table 2). As its design was more or less equivalent to the control group in the blocking design, we will focus on this experiment and explain it in greater detail. During training, participants faced a standard HCL task, in which they had to learn to predict words that functioned as outcomes from other words that functioned as cues on a trial-by-trial basis. For example, on Trial AC-1, participants would see the words city and marble (cues A and $\mathrm{C}$ in this example). Then they would have to press the key of the first letter of Outcome 1, grass ${ }^{1}$. Pressing key "G" would be a correct response, whereas pressing any other key would be a mistake. After pressing a key, participants were shown the correct word and received feedback on their response. These stimuli worked functionally exactly in the same way as those used in HCL tasks like the allergy task described before. Some researchers might consider this task as an atypical HCL task because stimuli are not framed within a causal scenario. However, there are two reasons to undermine this worry. First, there are numerous examples of studies using non-causal learning tasks that have been referred to as HCL tasks. For example, some studies focused on generalization and discrimination are not based on causal

\footnotetext{
${ }^{1}$ Although the words were Spanish words, we will continue using the same employed by McKoon and Ratcliff (1979) in the examples for the sake of simplicity.
}

learning tasks $[63,64]$. Second, our task strictly conforms to the most extended definitions of HCL (e.g., [62, p.289]).

Cue-outcome pairings were arranged according to the blocking design shown in Table 3. After training had taken place, a priming recognition test was used to measure the associations formed. Thus, participants went through a series of trials in each of which a prime was followed by a target with a 300-ms SOA. Participants were required to determine, as soon as possible, whether the target word had appeared during the training phase or not. In $50 \%$ of the trials, target words were old words that had been previously presented during the training phase. In such cases, a positive response should be made by pressing the left (or right for half of the participants) mouse button. In the remaining trials, a negative response should be made by pressing the alternative mouse button. Both old and new target words consisted of names from the same category of objects and were controlled for frequency and length. Also, the role of words as old or new was counterbalanced across participants. Primetarget pairs in old-target trials were arranged according to three different conditions, though only two of them are relevant here. In a coherent condition, prime-target pairs were consistent with the relationships learned during training. For example, marble would serve as the prime and grass as the target. In a recombined condition, each prime-target pair was made up of a cue and an unrelated outcome from the training phase (see Table 3). As an example, the words lovely and grass would serve as the prime and the target, respectively. Since lovely had been paired with a word different than grass during training, it would serve as a control condition. Using the appropriate controls and counterbalances, primes gave no information about what word would appear as the target, or about what response should be given on each trial. Note, as well, that participants did not need to use the relationships learned during training to respond during this test, and that they were instructed to respond only to the target and to read but ignore the prime. Also, the proportion of trials on which the prime had been associated during training with the target was only one out of six. All of this together with the SOA used made the engagement of high order processes very unlikely.

Table 3. Design of blocking experiment by Morís et al. [54]

\begin{tabular}{|c|c|c|c|}
\hline \multicolumn{2}{|c|}{ Training } & \multicolumn{2}{c|}{ Test } \\
\hline Phase 1 & Phase 2 & Coherent & Recombined \\
\hline \hline & $12 \times \mathrm{AC} 1$ & $\mathrm{C}-1 ?$ & $\mathrm{D}-1 ?$ \\
\hline $12 \times \mathrm{A} 1$ & $12 \times \mathrm{xD} 2$ & $\mathrm{D}-2 ?$ & $\mathrm{E}-2 ?$ \\
\hline $12 \times \mathrm{x} 2$ & $12 \times \mathrm{EF} 3$ & $\mathrm{E}-3 ?$ & $\mathrm{G}-3 ?$ \\
\hline & $12 \times \mathrm{XH} 4$ & $\mathrm{G}-4 ?$ & $\mathrm{C}-4 ?$ \\
\hline
\end{tabular}

Note: Only the critical test trials are presented. The whole set of test trials ensured that primes (cues) gave no information about what target stimulus (outcome) would appear later and therefore about what response should be given. There were a total of 96 test trials, and the possible effect of outliers was controlled.

According to the design shown in Table $\mathbf{3}$, the blocked cues, C and D, were paired with their corresponding out- 
comes the same number of times as the overshadowing control cues, E, F, G and H, were paired with their corresponding outcomes. However, Cues C and D were always present in compound with Cues $\mathrm{A}$ and $\mathrm{B}$, respectively, which had been established as perfect predictors of Outcomes 1 and 2, respectively, in a previous training phase, rendering Cues $\mathrm{C}$ and $\mathrm{D}$ uninformative. As for the control cues, all of them had a significant informative value because the accompanying cues did not have enough informative value on their own to render the other constituent of the compound uninformative. According to associative theories of blocking, the learning mechanism somehow calculates the informative value of cues to predict the outcome, which, in turn, determines the amount of activation spreading from the representation of the cue to the representation of the outcome when the cue is presented. Consequently, the amount of activation spreading from the representation of the blocked cues to the representation of their respective outcomes should be less than the amount of activation spreading from the representation of the control cues to the representation of their respective outcomes. In other words, the recognition priming effect for the control cues should be greater than for the blocked cues.

Although it is not easy to derive a clear prediction from non-associative theories of learning, it is interesting to note that some influential proposals have explained blocking as the result of inferences or of the application of statistical rules that would depend on control and effortful processes that would take place at test (e.g. [26]). For these processes to perform effectively, the information should be stored in a rather raw manner as it is assumed to occur according to some episodic memory models. Thus, the associative memories corresponding to the experienced events throughout the training trials should reflect, more or less, the contents of the different trial types. This would explain why participants seem to use the information stored in different ways depending on the type of question used at test or why concurrent memory-loading tasks seem to hinder the blocking effect especially when they are performed at test. From this perspective, no difference should be found between the blocking and the control cues regarding the recognition priming effect.

Morís et al.'s [54] results showed shorter response times for coherent (trials E-3 and G-4) than for recombined (trials G-3 and C-4) prime-target pairs in the overshadowing control condition. In other words, a priming effect was found in the control condition. However, no difference was found between response times for the coherent (trials C-1 and D-2) and for the recombined (trials D-1 and E-2) prime-target pairs in the blocking condition. Thus, no priming effect was found in the blocking condition. Moreover, the priming effect in the control condition was significantly greater than in the blocking condition.

These results are, therefore, fairly consistent with associative theories of learning. The recognition priming effect was sensitive to the relative predictive value of the cues to predict the outcomes, i.e., to the amount of information about the occurrence of the outcomes conveyed by the cues. The information stored in memory did not limit itself to reflecting the contents of the different trial types as in episodic memory. Rather, the associations measured through the recognition priming technique seemed to reflect the result of a learning process aimed at computing the predictive value of cues. Thus, this evidence supports the idea that the predictive relationships learned in HCL tasks are coded in associativebased representations, and that these representations could be, on their own, responsible for some of the blocking effects found in previous studies based on verbal judgments. Therefore, Morís et al.'s [54] results is consistent with the view that, regardless of the participation of inferential reasoning processes, associative processes could, after all, have some role in explaining HCL.

\section{DISCUSSION}

The present non-exhaustive review of previous results shows that associative repetition priming can be found after training in an HCL task. Moreover, we have shown that priming effects are sensitive to simple contingency manipulations and to the blocking treatment when a short SOA is used. As stated in the introduction, these results are precisely what would be expected if these HCL phenomena were produced by associative processes. Although the use of long SOAs allows for the intervention of high order strategic processes, short SOAs are known to preclude the operation of such processes. Thus, the only mechanisms expected to be involved in the retrieval of the information have to be fast, low level and automatic [37]. According to this, the evidence reviewed here gives strong support for an associative basis of, at least, some HCL phenomena. Therefore, it seems premature to discard associative theories as De Houwer [1] or Mitchell et al. [2] have recently proposed.

However, there is a limitation that needs to be pointed out. The results reviewed do not provide reliable information about what kind of mechanism is responsible for acquisition of the associative representations detected through the priming methodology. Reasoning or high level processes might have played an important role in forming associations by providing input to associative processes and by performing inferences and computations from the output of such processes (e.g. [55]). If this were the case, previous proposals (e.g. [5]) that accept that two kinds of mechanisms can be engaged during HCL and later on at test should be reexamined, and the interaction of these mechanisms would deserve further study. Knowing the conditions under which each of them operates and how they interact would allow the development of more general models of learning.

Another issue that remains to be solved is the extent to which the associative-based activation-spread mechanism is responsible for the different judgments and responses commonly used as dependent variables in ordinary blocking experiments conducted so far. On the one hand, according to the results found in our experiment, one could think that the design and procedure used in many previous blocking experiments meet the basic conditions to find blocking based on activation spread if a priming technique similar to ours had been used. But, on the other hand, it could be argued that, even if we take for granted that an associative process is at work in those cases, it could have little or null influence on verbal judgments, which, in turn, would rather rely on higher cognitive processes. Recall that the mere finding of blocking does not univocally point to an associative mechanism as the cause since evidence has been found that blocking can be produced by alternative higher cognitive proc- 
esses. This question becomes more complex if we consider that learning phenomena such as blocking has been found to depend on the type of judgment requested to participants [29]. Thus, associative-based activation spread could determine some verbal judgments more than others. Moreover, one could even reasonably think that there could be learning and test conditions affecting the extent to which associative mechanisms determine verbal judgments. We think that all of these are very interesting questions that could be investigated with the help of priming procedures like ours together with dissociation techniques.

Although beyond the scope of the present paper, it is important to note that there are theories of priming that are not based on the principle of activation spreading, and that, with appropriate assumptions, could be adapted to explain the findings reviewed here. Specifically, compound-cue theories (e.g. [56]) and episodic memory models (e.g. $[57,58])$ do not predict the blocking effect found by Morís et al. [54], but they are not necessarily incompatible with it. However, these types of theories, and all of those compatible with the results reviewed here, are based on associative principles of storage and retrieval and therefore compatible with associative theories of learning.

This review has been focused on associative repetition priming because of its potential as a measure of associative representations and retrieval processes, but other relevant evidence has been found using other procedures different from verbal judgements like IAT (standing for implicit association task) (e.g. [55]). Greenwald, McGhee and Schwartz [59] proposed the Implicit Association Test as a direct measure of memory associations. In this task, participants have to classify stimuli during two different conditions. In one of them participants are required to classify stimuli coherently with the associations that are supposed to exist between them. For example, tree and green should lead to responding with Key ' 1 ', whereas sky and blue should lead to responding with Key ' 2 '. In the incoherent condition, tree and blue should lead to responding with Key ' 1 ', whereas sky and green should lead to responding with Key ' 2 '. It is supposed that the difference in the classification reaction times reflects the influence of the existing associations. IAT has been recently used as a measure after an HCL task showing a potential interaction between reasoning and associative processes [55].

Finally, the use of this kind of priming methodology could also shed some light on further challenging phenomena like retrospective revaluation, which have also played an important role in the debate between associative and nonassociative researchers. For example, it could be interesting to know whether priming effects are sensitive to a backward blocking treatment. In a backward blocking design, participants are presented with A-1 pairings after having received AB-1 pairings, which eventually produces a decrease in the perceived relationship between $B$ and 1 (e.g. [60]). This sort of effect has led to the revision of previous associative models (e.g. $[11,61])$ and has constituted a central topic in HCL [3]. The study of the associations formed during retrospective revaluation experiments using priming techniques would allow us to know whether there is an associative basis for these learning phenomena or, alternatively, whether these effects are produced by inferential reasoning mechanisms.

\section{AUTHORS NOTE}

The research described here was supported by research grants from Junta de Andalucía (SEJ-406 and P.08SEJ-03586 SEJ) and Ministerio de Educación y Ciencia (ref. SEJ2007-63691/PSIC). Joaquín Morís has been supported by a F.P.D.I. fellowship also from Junta de Andalucía. The authors want to thank Francisco J. López for his commentaries of previous versions of this manuscript and collaboration. Correspondence concerning this article should be addressed to, Joaquín Morís, Departamento de Psicología Básica, Campus de Teatinos, Universidad de Málaga, Málaga 29071, Spain. Phone number: +34 952 132630/ Fax: +34 952132631 .

\section{REFERENCES}

[1] De Houwer J. The propositional approach to associative learning as an alternative for association formation models. Learn Behav 2009; 37: 1-20.

[2] Mitchell CJ, De Houwer J, Lovibond PF. The propositional nature of human associative learning. Behav Brain Sci 2009; 32: 183-246.

[3] Shanks, DR. Learning: from association to cognition. Annu Rev Psychol 2010; 61: 273-301.

[4] Shanks DR. Associationism and cognition: Human contingency learning at 25. Q J Exp Psychol 2007; 60:291-309.

[5] Dickinson A. Causal learning: an associative analysis. Q J Exp Psychol 2001; 54B: 3-25.

[6] Dickinson A, Shanks DR, Evenden JL. Judgement of act-outcome contingency: the role of selective attribution. Q J Exp Psychol 1984; 36A: 29-50.

[7] Shanks DR. Forward and backward blocking in human contingency judgement. Q J Exp Psychol 1985; 37B: 1-21.

[8] Rescorla RA, Wagner AR. A theory of Pavlovian conditioning: Variations in the effectiveness of reinforcement and nonreinforcement. In: Black AH, Prokasy WF, Eds. Classical conditioning II: Current research and theory USA: Appleton-Century-Crofts 1972; pp. 64-99.

[9] Rescorla RA. Probability of shock in the presence and absence of CS in fear conditioning. J Comp Physiol Psychol 1968; 66: 1-5.

[10] Cobos PL, López FJ, Caño A, Almaraz J, Shanks DR. Mechanisms of predictive and diagnostic causal induction. J Exp Psychol: Anim Behav Process 2002; 28: 331-46.

[11] Dickinson A, Burke J. Within-compound associations mediate the retrospective revaluation of causality judgements. Q J Exp Psychol 1996; 49B: 60-80.

[12] Karazinov DM, Boakes RA. Second order conditioning in human predictive judgments when there is little time to think. Q J Exp Psychol 2007; 60: 44-60.

[13] Le Pelley ME, McLaren IPL. Retrospective revaluation in humans: Learning or memory? Q J Exp Psychol 2001; 54B: 311-52.

[14] Wagner AR. SOP: a model of automatic memory processing in animal behavior. In: Spear NE, Miller RR, Eds. Information processing in animals: Memory mechanisms. USA: Lawrence Erlbaum Associates, Inc 1981; pp. 5-47.

[15] McLaren IPL, Mackintosh NJ. An elemental model of associative learning: I. Latent inhibition and perceptual learning. Anim Learn Behav 2000; 28: 211-46.

[16] Cheng PW, Novick LR. Covariation in natural causal induction. Psychol Rev 1992; 99: 365-382.

[17] De Houwer J, Beckers T, Vandorpe S. Evidence for the role of higher-order reasoning processes in cue competition and other learning phenomena. Learn Behav 2005; 33: 239-49.

[18] Kamin LJ. "Attention-like" processes in classical conditioning. In: Jones MR, Ed. Miami symposium on the prediction of behavior, 1967: Aversive stimulation. USA: University of Miami Press 1968; pp. 9-31.

[19] Lovibond PF, Been SL, Mitchell CJ, Bouton ME, Frohardt R. Forward and backward blocking of causal judgment is enhanced by additivity of effect magnitude. Mem Cogn 2003; 31: 133-42.

[20] Beckers T, De Houwer J, Pineño O, Miller RR. Outcome additivity and outcome maximality influence cue competition in human causal learning. J Exp Psychol: Learn Mem Cogn 2005; 31: 23849. 
[21] Beckers T, Miller RR, De Houwer J, Urushihara K. Reasoning rats: forward blocking in Pavlovian animal conditioning is sensitive to constraints of causal inference. J Exp Psychol: Gen 2006; 135: $92-$ 102.

[22] De Houwer J, Beckers T, Glautier S. Outcome and cue properties modulate blocking. Q J Exp Psychol 2002; 55A: 965-85.

[23] Mitchell CJ, Lovibond PF. Backward and forward blocking in human electrodermal conditioning: Blocking requires an assumption of outcome additivity. Q J Exp Psychol 2002; 55B: 311-29.

[24] Mitchell CJ, Lovibond PF, Condoleon M. Evidence for flexible rule use in blocking of causal judgments. Learn Motiv 2005; 36: 77-87.

[25] Schmajuk N, Larrauri J. Associative models can describe both causal learning and conditioning. Behav Process 2008; 77: 44345.

[26] De Houwer J, Beckers T. Secondary task difficulty modulates forward blocking in human contingency learning. Q J Exp Psychol 2003; 56B: 345-57.

[27] Vandorpe S, De Houwer J, Beckers T. Further evidence for the role of inferential reasoning in forward blocking. Mem Cogn 2005; 33: 1047-56.

[28] Price PC, Yates JF. Associative and rule-based accounts of cue interaction in contingency judgment. J Exp Psychol: Learn Mem Cogn 1995; 21: 1639-55.

[29] Matute H, Arcediano F, Miller RR. Test question modulates cue competition between causes and between effects. J Exp Psychol: Learn Mem Cogn 1996; 22: 182-96.

[30] Matute H, Vegas S, De Marez PJ. Flexible use of recent information in causal and predictive judgments. J Exp Psychol: Learn Mem Cogn 2002; 28: 714-25.

[31] Pineño O, Denniston JC, Beckers T, Matute H, Miller RR. Contrasting predictive and causal values of predictors and of causes. Learn Behav 2005; 33: 184-96.

[32] Vadillo MA, Miller RR, Matute H. Causal and predictive-value judgments, but not predictions, are based on cue-outcome contingency. Learn Behav 2005; 33: 172-83.

[33] Lovibond PF. Causal beliefs and conditioned responses: retrospective revaluation induced by experience and by instruction. J Exp Psychol: Learn Mem Cogn 2003; 29: 97-106.

[34] Koivisto M. Time course of semantic activation in cerebral hemispheres. Neuropsychologia 1997; 35: 447-504.

[35] Neely JH. Semantic priming and retrieval from lexical memory: Evidence for facilitatory and inhibitory processes. Mem Cogn 1976; 4: 648-54.

[36] Neely JH. Semantic priming effects in visual word recognition: A selective review of correct findings and theory. In: Besner D, Humphreys GW, Eds. Basic processing in reading: Visual word recognition, Erlbaum 1991; pp. 264-336.

[37] Zeelenberg R, Pecher D, Raaijmakers JGW. Associative repetition priming: A selective review and theoretical implications. In: Bowers $\mathrm{J}$ and Marsolek C, Eds. Rethinking implicit memory. Oxford: Oxford University Press 2003; pp. 261-283.

[38] Perea M, Gotor A. Associative and semantic priming effects occur at very short stimulus-onset asynchronies in lexical decision and naming. Cognition 1997; 62: 223-40.

[39] Hutchinson KA. Is semantic priming due to association strength or feature overlap? a microanalytic review. Psychon Bull Rev 2003; 10: 785-813.

[40] Graf P, Schacter DL. Selective effects of interference on implicit and explicit memory for new associations. J Exp Psychol: Learn Mem Cogn 1987; 32: 475-90.
[41] McKoon G, Ratcliff R. Priming in episodic and semantic memory. J Verb Learn Verb Behav 1979; 18: 463-80.

[42] Pecher D, Raaijmakers JGW. Automatic priming for new associations in lexical decision and perceptual identification. Q J Exp Psychol 1999; 52A: 593-614.

[43] Pylkkänen L, Marantz A. Tracking the time course of word recognition with MEG. Trends Cogn Sci 2003; 7: 187-89.

[44] Anderson JR. A spreading activation theory of memory. J Verb Learn Verb Behav 1983; 22: 261-95.

[45] Collins AM, Loftus EF. A spreading activation theory of semantic memory. Psychol Rev 1975; 82: 407-28.

[46] Neely JH. Semantic priming and retrieval from lexical memory: Roles of inhibitionless spreading activation and limited-capacity attention. J Exp Psychol: Gen 1977; 106: 226-54.

[47] McNamara TP. Priming and constraints it places on theories of memory and retrieval. Psychol Rev 1992; 99: 650-62.

[48] Posner MI, Snyder CR. Attention and cognitive control. In: Solso RL, Ed. Information processing and cognition. USA: Erlbaum 1975; pp. 55-85.

[49] Meyer D, Schvaneveldt R. Meaning, memory and mental processes. Science 1976; 192: 27-33.

[50] Watkins MJ, Tulving E. Episodic memory: When recognition fails. J Exp Psychol: Gen 1975; 104: 5-38.

[51] Carroll M, Kirsner K. Context and repetition effects in lexical decision and recognition memory. J Verb Learn Verb Behav 1982; 21: 55-69.

[52] Durgunoglu AY, Neely JH. On obtaining episodic priming in a lexical decision task following paired-associate learning. J Exp Psychol: Learn Mem Cogn 1987; 13: 206-22.

[53] McKoon G, Ratcliff R. Automatic activation of episodic information in a semantic memory task. J Exp Psychol: Learn Mem Cogn 1986; 12: 108-15.

[54] Morís J, Luque D, Cobos PL, López FJ. Associative learning phenomena and associative repetition priming. In preparation.

[55] De Houwer J, Vandorpe S. Using the Implicit Association Test as a measure of causal learning does not eliminate effects of rule learning. Exp Psychol 2010; 57(1): 61-7.

[56] Ratcliff R, McKoon G. A retrieval theory of priming in memory. Psychol Rev 1988; 95: 385-408.

[57] Raaijmakers JGW, Shiffrin RM. Search of associative memory. Psychol Rev 1981; 88: 93-134.

[58] Hintzman DL. "Schema abstraction" in a multiple-trace memory model. Psychol Rev 1986; 93: 411-28.

[59] Greenwald AG, McGhee DE, Schwartz JLK. Measuring individual differences in implicit cognition: The Implicit Association Test. J Pers Soc Psychol 1998; 74: 1464-80.

[60] Wasserman EA, Berglan LR. Backward blocking and recovery from overshadowing in human causal judgment: the role of withincompound associations. Q J Exp Psychol 1998; 51B: 121-38.

[61] Van Hamme LJ, Wasserman EA. Cue competition in causality judgments: The role of nonpresentation of compound stimulus elements. Learn Motiv 1994; 25: 127-51.

[62] De Houwer J, Beckers T. A review of recent developments in research and theories on human contingency learning. Q J Exp Psychol 2002; 55B: 289-310.

[63] Torwart A, Lachnit H. Symmetrical generalization decrements: Configural stimulus processing in human contingency learning. Learn Behav 2009; 37: 107-15.

[64] Vervliet B, Vansteenwegen D, Eelen E. Generalization gradients for acquisition and extinction in human contingency learning. Exp Psychol 2006; 53: 132-42. 